\title{
The Molecular Cloning and Characterization of Potential Chick DM-GRASP Homologs in Zebrafish and Mouse
}

\author{
John P. Kanki, ${ }^{1, *}$, Susannah Chang, $^{2}$ and John Y. Kuwada ${ }^{1}$ \\ ${ }^{1}$ Department of Biology, University of Michigan, Ann Arbor, Michigan 48109-1048; and ${ }^{2}$ Department of \\ Anatomy, University of Pennsylvania School of Medicine, Philadelphia, Pennsylvania 19104
}

\section{SUMMARY}

A full-length zebrafish cDNA clone and a partial mouse cDNA clone similar to chick $D M-G R A S P$ were isolated and analyzed. The nucleotide sequence of the full-length zebrafish clone shares $54 \%$ identity, and predicts $39 \%$ amino acid identity, with chick DM-GRASP. The partial mouse clone shares $76 \%$ nucleotide identity, and predicts $76 \%$ amino acid identity, with chick DM-GRASP. The predicted proteins encoded by both of these clones exhibit conserved structural domains that are characteristic of the chick protein. These features may identify them as a distinct subfamily within the immunoglobulin superfamily of cell adhesion molecules. Expression of the zebrafish DM-GRASP protein is similar to chick DMGRASP and is principally restricted to a small subset of developing sensory and motor neurons during axonogenesis. Zebrafish DM-GRASP expression was temporally regulated and limited to specific axon domains. This regional expression correlated with fasciculated axon domains. These results suggest that the zebrafish and mouse cDNA clones represent the respective fish and mammalian homologs of chick DM-GRASP. The highly selective expression of zebrafish DM-GRASP suggests that it is involved in the selective fasciculation and guidance of axons along their normal pathways. (c) $1994 \mathrm{John}$ Wiley \& Sons, Inc.

Keywords: zebrafish, DM-GRASP, Ig superfamily, axon guidance, fasciculation.

\section{INTRODUCTION}

The nervous system develops and functions normally because neural growth cones find and establish appropriate connections with specific targets. Specific connections are formed early during embryogenesis by axons following stereotyped pathways to their synaptic targets. Growth cones follow stereotyped patterns of axonal outgrowth by recognizing cues that distinguish their proper pathways from inappropriate ones. Growth cones respond to a variety of attractive and inhibitory signals in a

Received January 11, 1994; accepted February 16, 1994

Journal of Neurobiology, Vol. 25, No. 7, pp. 831-845 (1994)

(C) 1994 John Wiley \& Sons, Inc.

CCC 0022-3034/94/070831-15

* To whom correspondence should be addressed.

${ }^{\dagger}$ Present address: Department of Molecular Biology, Moffett Laboratory. Princeton University, Princeton, NJ 08544. complex and changing environment (e.g., Bentley and Caudy, 1983; Raper et al., 1983; Tosney and Landmesser, 1985; Bastiani et al., 1986; Kap fhammer and Raper, 1987; Kuwada, 1986; Harris et al., 1987; Dodd and Jessell, 1988). These signals may include cell surface proteins (Rathjen et al., 1987), extracellular matrix proteins (Reichardt and Tomaselli, 1991), and diffusable factors (Lumsden and Davies, 1991; Tessier-Lavigne and Placzek, 1991). Molecules whose expression is restricted to specific substrates or axons are of particular interest since they represent potential cues for guiding growth cones along their pathways.

Cell surface molecules that are expressed by subsets of developing axons have been identified in a variety of embryos. Many of these molecules are members of the immunoglobulin (Ig) superfamily of adhesion proteins (Williams and Barclay, 1988). These proteins mediate specific cell adhe- 
sion interactions that, in the case of neurons, may influence selective adhesion to substrates along a specific axon pathway. Some of the fasciclins are well-characterized members of the Ig superfamily which are expressed on specific axonal bundles in insects. Genetic and antibody perturbation experiments suggest that fasciclin II is necessary for growth cones to fasciculate and extend upon specific axon bundles during development (Harrelson and Goodman, 1988; Grenningloh et al., 1990). In vertebrates a number of putative axon guidance molecules have also been characterized (Rathjen and Jessell, 1991). Some of these molecules are expressed selectively on subsets of axons. One intriguing molecule is DM-GRASP (also called SC1 and BEN) which is a member of the Ig superfamily and is principally expressed on developing sensory and motor axons in the chick (Tanaka and Obata, 1984; Pourquie et al., 1990; Burns et al., 1991; ElDeeb et al., 1992). It exhibits homophilic binding and promotes neurite extension in vitro suggesting that it participates in selective axon fasciculation and axon guidance (Burns et al., 1991; Tanaka et al., 1991; Pourquie et al., 1992).

In addition to being expressed on selective subsets of neurons, these molecules may also be regionally expressed on specific axon domains. For example, TAG-1 and $\mathrm{Ll}$ are expressed in a domain-specific pattern on the axons of commissural neurons in the rat spinal cord (Dodd et al., 1988). Their expression is spatially regulated such that TAG-1 is expressed on the ipsilateral, circumferential portion of the axons as they run towards the floorplate at the ventral midline. Once the commissural axons pass the floorplate to the contralateral side they turn, run anteriorly, and express L1. The switch from TAG-1 to L1 expression suggests that these molecules may be responsible for guiding the growth cones along different regions of their pathway.

We have cloned a zebrafish cDNA similar to chick DM-GRASP in order to examine the expression of DM-GRASP in the well-characterized nervous system of the zebrafish embryo. The zebrafish is an attractive vertebrate system for in vivo analysis of early development (Eisen, 1991; Weinberg, 1992 ). During early embryonic stages it is possible to examine the development of individual neurons as they project their axons and establish highly stereotyped pathways (e.g., Kimmel et al., 1982; Myers, 1985; Eisen et al., 1986; Kuwada and Bernhardt, 1990). The well-characterized development of these identified neurons makes it possible to distinguish regional differences in the expression of molecules on their axonal domains. Thus the zebrafish embryo is an attractive system for the detailed examination of the expression of putative axon guidance cues such as DM-GRASP. The detailed analysis of the developmental expression of such molecules on these neurons may elucidate their potential roles in directing axonal outgrowth in vivo.

We have cloned potential homologs of DMGRASP in the mouse and the zebrafish and have examined DM-GRASP expression on identified neurons in the early zebrafish embryo. Our results indicate that this molecule is selectively expressed on a small subset of axon tracts, and that it is regionally expressed on specific axon domains. The selectivity of DM-GRASP expression and its differential expression on specific axon domains may regulate their ability to fasciculate with each other and thereby determine the pattern of their axonal trajectories.

\section{METHODS}

\section{Sequencing and Analysis}

A $\lambda$ gt 11 cDNA library was constructed from total RNA isolated from 32- to 48-h zebrafish embryos (K. Zinn). Approximately $6 \times 10^{5}$ plaques were screened using established procedures for lowstringency hybridization screening (Sambrook et al., 1989). Duplicate filter lifts were probed with a ${ }^{32} \mathrm{P}$-random-primed fragment $(1.6 \mathrm{~kb})$ of the chick DM-GRASP clone. Three hybridizing plaques were isolated and their cDNA inserts were subcloned into Bluescript $\mathrm{SK}^{-}$(Stratagene) and sequenced using the dideoxy chain termination method (Sanger et al., 1977) with Sequenase (USB). Sequence alignments to Genbank using the FASTA program (ktup $=6$, Pearson and Lipman, 1988) were performed and further nucleotide and amino acid analysis was done using the MacVector (IBI) and GeneWorks (IntelliGenetics) programs. Protein alignments were performed using a PAM-250 scoring matrix. Two additional clones were isolated under conditions of high stringency from a $\lambda$ ZAP library (Stratagene) made from 20 to 28-h zebrafish embryos (generously supplied by D. J. Grunwald ), using a ${ }^{32}$ P-random-primed fragment $(1.6 \mathrm{~kb})$ of the zebrafish DM-GRASP clone as probe. These duplicate clones were isolated from the $\lambda \mathrm{ZAP}$ phage and sequenced as described above.

To obtain the mouse cDNA clone, $10^{7}$ plaques 
from an adult mouse brain cDNA library (Clontech) were screened using a ${ }^{32} \mathrm{P}$-random-primed fragment $(1.5 \mathrm{~kb})$ of the chick DM-GRASP clone. Seven hybridizing plaques were isolated using standard conditions for low-stringency hybridization. After the cDNA inserts were subcloned, one was sequenced and further analyzed as described above.

\section{Generation of Glutathione S-Transferase Fusion Proteins and Polyclonal Antisera}

The polymerase chain reaction (PCR) (Saiki et al., 1988 ) was used to amplify an 812-bp sequence from the 5 ' end of the zebrafish DM-GRASP clone with oligonucleotides containing Not $1\left(5^{\prime}\right)$ and Smal (3') linkers. The amplified fragment corresponded to zebrafish DM-GRASP nucleotides 160-972 encoding amino acids 1-271. The PCR fragment was isolated using TA cloning (Invitrogen ), and then digested with Not 1 and Smal. This fragment was inserted into pGEX-KN (Hakes and Dixon, 1992) which had been digested with $E c o$ R 1, end-filled with the Klenow fragment of DNA polymerase I, and digested with Not 1 . This placed the zebrafish DM-GRASP amino acid sequence in-frame behind the glutathione S-transferase sequence. The fusion protein was expressed in Escherichia Coli, and purified from the bacterial extracts by affinity chromatography with glutathione-sepharose (Pharmacia) (Smith and Johnson, 1988). The purified zebrafish DM-GRASP fusion protein was used for the production of antisera. Then, $50 \mu \mathrm{g}$ of protein was injected at 4-week intervals into three rabbits (UM5, UM27, and UM28) (Cocalico Biologicals). Preimmune serum was obtained from each rabbit prior to immunization with the antigen. After immunization, antiserum was collected every 2 weeks. The sera were tested for reactivity by immunoblot analysis using bacterial lysates containing the fusion protein. Antiserum from UM 5 and pooled antisera from UM 27 and UM28 were affinity purified using established methods (Harlow and Lane 1988) on a column containing the zebrafish fusion protein covalently coupled to CNBr-activated sepharose (Pharmacia).

\section{Immunoblot Analysis and Immunocytochemistry}

Zebrafish embryos were collected from a laboratory breeding colony, maintained at $28.5^{\circ} \mathrm{C}$, and developmentally staged according to procedures described in Myers et al. (1986).

For immunoblot analysis embryos were collected at various stages, deyolked if necessary, and lysed ( $2-\mu$ l lysis buffer/embryo) in a $20-\mathrm{m} M$ Phosphate buffer ( $\mathrm{pH} 7.5$ ) containing $100 \mathrm{~m} \mathrm{M} \mathrm{NaCl}$, $10 \mathrm{~m} M$ EDTA, $1 \%$ sucrose, $1 \%$ NP40, $1 \mathrm{~m} M$ PMSF, $1 \mu \mathrm{g} / \mathrm{ml}$ aprotinin and leupeptin. The lysates were centrifuged briefly, and an equal volume of 3\% SDS reducing gel sample buffer was added to the supernatant. Lysates containing the bacterial fusion protein were prepared by adding sample buffer directly to bacterial pellets. The lysates were fractionated by SDS-PAGE on $8 \%$ or $10 \%$ gels. Proteins were either stained with Coomassie blue or transferred to nitrocellulose filters. The filters were blocked in Tris-buffered saline containing 1\% BSA and probed with antiserum at 1:250-1000 dilution, followed by ${ }^{125}$ I-Protein A (Amersham). Blots were exposed to Kodak X-OMAT film with intensifying screens at $-70^{\circ} \mathrm{C}$.

For immunocytochemical analysis, embryos were collected between $12 \mathrm{~h}$ and 5 days postfertilization, fixed in $4 \%$ paraformaldehyde in $0.1 \mathrm{M}$ phosphate buffer ( $\mathrm{pH} \mathrm{7.4)}$ ) for $6-18 \mathrm{~h}$ at $4^{\circ} \mathrm{C}$ and subsequently processed as whole-mount preparations or cryostat frozen sections as described in the Zebrafish Book (ed. Westerfield, 1993). For frozen sections embryos were embedded in $1.5 \%$ agar $/ 5 \%$ sucrose, cryoprotected in $30 \%$ sucrose, and frozen in OCT compound. Sections $(20 \mu \mathrm{m})$ were cut on a Reichert-Jung 2800 cryostat. Whole-mounted embryos and sections were incubated with primary antisera (dilutions ranged from 1:10-1000) for $16-24 \mathrm{~h}$ at $4^{\circ} \mathrm{C}$. Slides with frozen sections were maintained in humidified chambers. Primary antibodies were detected with a diaminobenzidine (DAB) reaction product after incubating with a biotinylated anti-rabbit secondary antibody followed by a biotin/avidin peroxidase complex (Vectastain ABC, Vector Laboratories). Wholemounted embryos and sections were viewed and photographed with a Leitz Orthoplan 2 microscope using differential interference contrast optics.

\section{In Vitro Transcription and Translation}

PCR was used to amplify the zebrafish DMGRASP cDNA insert directly from the $\lambda$ GT11 clone using phage $\lambda$ primers. The amplified product was isolated using TA cloning (Invitrogen). Zebrafish DM-GRASP mRNA was transcribed in vitro using SP6 polymerase, and was isolated by phenol/chloroform extraction followed by ethanol 
precipitation (Sambrook et al., 1989). The mRNA was then translated in vitro using a rabbit reticulocyte lysate (Promega) in the presence of ${ }^{35} \mathrm{~S}$-labeled methionine (Amersham). Translations were performed in the presence and absence of canine pancreatic microsomal membranes (Promega). The translation products were analyzed by SDS-PAGE on $8 \%$ gels followed by fluorography with Enlightening (Dupont). Flourographed gels were exposed to Kodak X-OMAT film at $-70^{\circ} \mathrm{C}$.

\section{RESULTS}

\section{Isolation and Analysis of a Zebrafish DM-GRASP cDNA Clone}

A $1.6-\mathrm{kb}$ fragment of chick $D M-G R A S P$ was used to screen an oligo(dT)-primed zebrafish cDNA library made from 32 - to 48 -h embryos. Three positive plaques were isolated and subcloned, the largest of these contained an insert of 2070 base pairs. Sequence analysis revealed an open reading frame of 1692 bp with a $159 \mathrm{bp}$ leader and 218 bp of $3^{\prime}$ untranslated sequence (Fig. 1). The open reading frame predicts a precursor protein of 564 amino acids including a putative 22 amino acid signal sequence (von Heijne, 1986) that directs protein translation to the endoplasmic reticulum (ER). A developmental Northern blot of total RNA extracted from zebrafish embryos between 24 and 74 $\mathrm{h}$ was probed with the zebrafish cDNA clone and revealed a single $3.6 \mathrm{~kb}$ mRNA transcript (data not shown). This differs from the chick where two apparent mRNA transcripts encoding DM-GRASP have been described (Tanaka et al., 1991).

Genbank searches with the FASTA DNA alignment program revealed that the zebrafish cDNA shared 54\% identity with the chick DM-GRASP nucleotide sequence. The predicted zebrafish DMGRASP protein, like the chick, also showed lower homology (20\%) to another member of the Ig superfamily, the human metastatic melanoma protein MUC18 (Lehmann et al., 1989; Burns et al., 1991). A Kyte-Doolitle hydrophilicity plot of the predicted zebrafish DM-GRASP protein [Fig. $2(A)]$ showed a hydrophobic region at the amino terminus of the protein, indicative of a signal sequence. An additional hydrophobic region near the carboxyl end of the protein predicted a 22 amino acid transmembrane domain from amino acids $507-529$, that is followed by a hydrophilic cytoplasmic tail. Four potential asparagine-linked glycosylation sites are present at amino acids 92,
350,441 , and 465; all of these are located in the predicted extracellular domain.

Alignment of chick DM-GRASP and the predicted amino acid sequence of the zebrafish clone demonstrates that the proteins share $39 \%$ amino acid identity [Fig. 2(B)]. The highest regions of amino acid homology were the transmembrane (64\%) and cytoplasmic ( $53 \%$ ) domains. When the predicted signal sequences of zebrafish and chick DM-GRASP proteins are cleaved, the resulting mature core proteins begin at homologous leucine residues and have similar predicted molecular weights ( 59 and $62 \mathrm{kD}$, respectively). The zebrafish and chick DM-GRASP proteins contain conserved structural domains classifying them as members of the Ig superfamily of cell adhesion molecules. Ig domains are characterized by conserved amino acid sequences that typically, but not always, have two cysteine residues that stabilize their globular tertiary structure through intrachain disulfide bonds (Williams and Barclay, 1988). They are composed of folded $\beta$ sheets, and are classified into categories depending upon conserved amino acid sequences surrounding the cysteine residues, and the length of the domains. V-type domains usually have 65-75 amino acids between cysteine residues, while C-type domains generally have 50-60. Most neural cell adhesion molecules of the Ig family contain C2-type domains, often followed by fibronectin type III domains. Chick DM-GRASP is rare in having two $V$-type Ig domains followed by three C2-type Ig domains. This VVCCC Ig domain sequence may be unique to DM-GRASP and the MUC18 protein (Burns et al., 1991; Lehmann et al., 1989). The alignment of the zebrafish clone and chick DM-GRASP by Ig domains (labeled I$\mathrm{V}$ ) demonstrates that the rare VVCCC Ig domain structure is also found in the zebrafish DMGRASP clone [Figs. 2(B and C)].

One discrepancy between the conserved Ig domains of the chick and zebrafish DM-GRASP clones is located in the fourth Ig domain. A leucine residue (at position 347) substitutes for the first cysteine of this domain in the zebrafish clone. To be certain that the leucine/cysteine substitution was not due to a sequence anomaly of the $\lambda \mathrm{GT} 11$ clone, two additional cDNA clones were isolated by high-stringency hybridization screening of a different zebrafish cDNA library ( $\lambda Z \mathrm{ZAP}$ ) using the $\lambda \mathrm{GT} 11$ clone as a probe. Sequence analysis of these additional clones confirmed the predicted leucine residue in fourth Ig domain. Although cysteine residues are known to stabilize the Ig domains, they 
CCG TCT AAG CTC CAG ACT CCA CCT GCG CTC TGT AGA GCA ACC CGT CTE ATC TTT CAG CGC ACT CAA CTC TCA ATA CTA TCT AAC AAC TTC

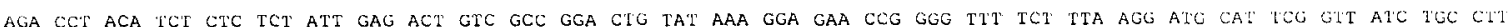
Mot hig Sor Val Ilo Cys Lou

TTC GGT GCC TTC ATA GCA GCC GCT' TTG TTT GCT CCA GGG AGC TGC CTG CCG ACG GTT ATA GGT CTG TAC GGT GAG ACC ATC GAA GTG CCA

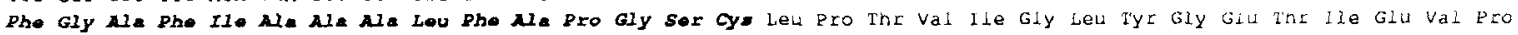

TGC AAC AAT GGA AAT AAC AAG CCA GAT GGC CTT ATT TTC ACC AAA TGG AAA TAT GCA AAA GAT GAC GGC TCC CCT GGC GAT CTA CTA ATA Cys Asn Asn Gly Asn Asn lys pro Asp Gly leu Ile phe thr lys Trp lys tyr Ala lys Asp Asp Gly Ser pro Gly Asp leu Leu Ile AAG CAG GCA CAG AAA GAT GAT CCG ACT GTG TCT GCT ATG GAC GGC TAC AAA ACT AGA GTT AGC ATC GCT GCT AAT TCC AGC TTA CTG ATT Lys Gin Ala Gln lys Asp Asp pro thr val ser Ala Met Asp Gly Tyr lys thr Arg val ser Ile Ala Ala Asn ser ser Leu Leu Ile

GCC CAG GGC TCT TTG ACT GAC CAA AGA GTC TTC ACC TGC ATG GTG GTG TCT TCA ACT AAT CTG GAG GAA TTC TCT GTG GAA GTT AAA GTT

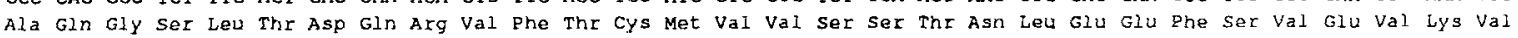
CAC AAA AAA CCA TCA GCC CCT GTA ATC AAA AAC AAA GTG AAA GAA CTG GAA AAT GGC AAA CTG ACG CAG TTG GGG GAA TGT GTG GTG GAG His Lys lys pro ser Ala pro val Ile Lys Asn lys val lys Glu Leu glu Asn Gly lys leu thr gin Leu Gly Glu Cys Val Val Glu

AGC GCC AAC CCA GCA GCA GAT GTC ATC TGG ATG AAG AAC AAC CAG GCT GTG GTG GAT GAT GGC AAG ACG ATC ATI ATC ACA TCA GAT GTC Ser Ala Asn pro Ala Ala Asp Leu Ile trp Mer Lys Asn Asn Gln Ala Leu val Asp Asp Gly Lys thr Ile Ile Ile Thr ser Asp Val

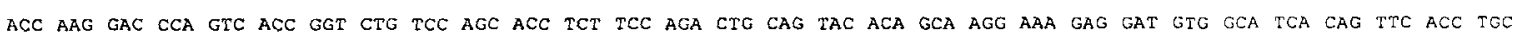
Thr Lys Asp pro val Thr Gly Leu Ser Ser Thr Ser ser Arg leu Gln Tyr Thr Ala Arg lys Glu Asp Val Ala ser Gln phe thr Cys

GTT GCA AAG CAC GTG ACG GGA CCC AAC CAG GTT TCA ACA CCC GAT ACC TTC CAA ATT CGC TAT CCC ACT GAG AAG GTG AGT CTA CAG GTT Val Ala Lys His Val Thr Gly pro Asn Gln Val Ser Thr pro Asp Thr phe Gin Ile Arg Tyr Pro Thr Glu Lys Val Ser Leu Gln Val

GTC TCT CAG AGC CCC ATT AGG GAA GGT GAT GAT GTG ACT CTG AAA TGC CAG GCG GAC GGA AAC CCT CCT CCT ACT AGC TTC AAC TTT AAC Val ser Gln ser pro Ile Arg Glu Giy Asp Asp Val Thr leu lys Cys Gln Ala Asp Gly Asn pro pro pro thr Ser phe Asn phe Asn

ATT AAG GGA AAG AAG GTC ACG GTG ACG GAC AAG GAT GTC TAC ACA CTG ACC GGC GTC ACC CGA GCC GAC AGC GGT GTG TAC AAG TGC TCT 108

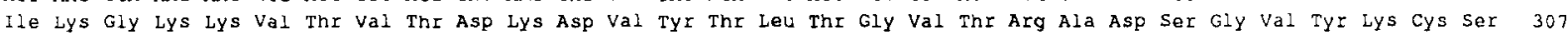

CTG CTT GAC AAC GAT GTG ATG GAG TCC ACT CAG ATC GTC ACA GTG AGC TTT CTG GAT GCA AGC CTC ACT CCT ACA GGC AAG GTG TTA AAA 1170 Leu Leu Asp Asn Asp val Met Glu ser thr Gin Ile val Thr Val ser phe leu Asp Ala Sex Leu Thr Pro thr Gly Lys val Leu Lys 337

AAG CTC GGG GAA AAC TTG GTA GTG TCT TTG GAG AAG AAT GCC TCT TCT GAA GTA AAA GTG ACG TGG ACT AAG GAT AAC CGT AAA CTG GAC 1260 Lys leu Gly Glu Asn Lel Val val Ser Leu Glu lys Asn Ala Ser Ser Glu Val lys Val Thr Trp Thr Lys Asp Asn Arg Lys Leu Asp 367

AAA CTG CCT GAT TTC TCC CAG TTG AGA TAC AGC GAC GCG GGC TTA TAC GTG TGT GAT GTG TCC ATT GAA GGA ATC AAA CAC AGC TTT TCC I350 Lys Leu pro Asp phe Ser Gln Leu Arg tyr Ser Asp Ala Gly Leu tyr val Cys Asp val ser Ile Glu Gly Ile Lys H1s ser phe Ser 397

TTC GAG CTT ACT GTG GAA GGT GGT CCA AGA ATT ACC GGC CTG ACA AAG CAT CGC AGC AAT GAC GGA AAA CAC aAA GTG TTG ACG TGC GAG 1440 Phe Glu Leu Thr Val Glu Gly Gly Pro Arg Ile Thr Gly Leu Thr Lys H1s Arg Ser Asn Asp Gly Lys H1s Lys Val Leu Thr Gys Giu 427 GCA GAA GGT TCA CCT AAA CCI GAA GTG CAG TGG AGT GTC AAC GGA ACC GAC GAT GAA ACA TCG TAT GTC AAC GGA AAA GCC ACA TAC AAA 1530 Ala Glu Gly Ser Pro bys pro Glu Val Gin Trp ser Val Asn Gly Thr Asp Asp Glu Thr Ser Tyr Val Asn Gly Lys Ala thr Tyr Lys 457 CTG ACG GTG GTC CCG AGT AAG AAC CTC ACC GTC AGC TGC CTC GTG ACC AAT AAA CTC GGT TTC GAC ACA AAG GAC ATC AGC GTG TTT TCC 1620

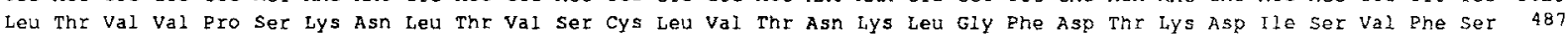

CTA TTT GAG GAG GAC AAG CCC AAA CCA GGA AAA AAT GAA GAT GGC GCA GAC CAA GCC AAA GTG ATT GTG GGT GTC GTG GTT GGA CTG TTT 1710 Leu Phe Glu Glu Asp lys pro lys pro Gly lys Asn Glu Asp Gly Ala Asp Gin Ala lys val ile val GIy val Val val Gly Leu phe 51 ?

CTA GCC GCT GCC CTG GTG GGA CTC ATC TAC TGG TTG TAT ATC AAG AAA ACA AGA CAA GGC AGC TGG AAG ACC GGA GAG AAG GAG ACT GGC 1800 Lel Ala Ala Ala Leu Val Gly leu rle Tyr Trp Leu Tyr Ile lys lys Thr Arg Gln Gly Ser Trp Lys Thr Gly Glu tys Glu Thr Gly 547

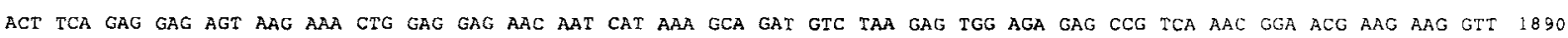
Thr Ser Glu Glu Ser lys Lys leu Glu Glu Asn Asn His Lys Ala Asp val

TGA ACC AGG AAA GAG GCC TTG TCA CAC TGA ATG TGC AGT ACT ACA CAC TTC GAT CTT CTT ATT CCA GAC ATC TGA TTC GAC GGC GAC GCC 1980 TCC TTC TGG CTG CTC GTG CGC ATA CCG CAT CGC AGA ATG TTT CCA AAA TAT ITC CTA CTC CAA ACA AAC AAT TTC CTA AAA AAA AAA AAG 2070

Figure 1 Nucleotide and predicted amino acid sequence of zebrafish DM-GRASP. The putative signal sequence is in bold italics, and the transmembrane domain is underscored. Asterisks denote potential asparagine-linked glycosylation sites. The zebrafish sequence has been registered with Genbank and given the following accession number: ZEFDMGRASP, L25273.

are not mandatory. A number of other molecules in the Ig superfamily are known to replace the cysteine residue with other hydrophobic residues, such as leucine, isoleucine, valine, tyrosine, or methionine (Williams and Barclay, 1988). Such substitutions are thought to be suitable for the maintenance and stabilization of the intrachain fold.

\section{Isolation and Analysis of a Mouse DM-GRASP cDNA Clone}

A $1.5-\mathrm{kb}$ fragment of chick DM-GRASP was used to screen a cDNA library from adult mouse brain (Clontech). Seven plaques were isolated and subcloned; one of these contained a 1288 bp cDNA 
A

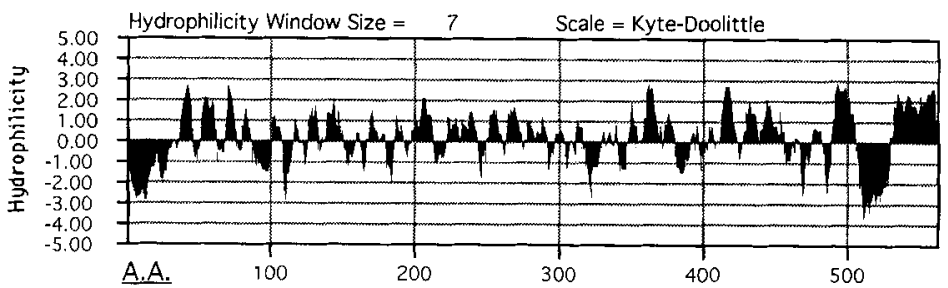

B

Zebrafigh DM-GRASP Chick DM-GRASP MOUBe DM-GRASP
¿-1 - - - -HSVI *- - - *FGAFI *AALF MEPPSRRRPASCRRR PLLCLLL ASLCM

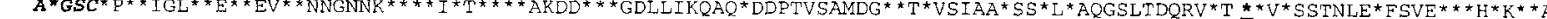
PPALGLYTVNAVYGDT I TMPCR - - LEVPDGLMFGKWKYEMPNGSPVFIAFRSSTKKNVQYDDVPDYKDRLSLSENYTLSIKNARIRHEKRFV CMMLVTEDDVSEEFTVVKVFKQPSQ

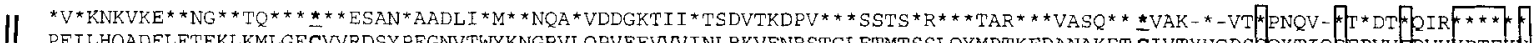
PEILHQADFLETEKLKMLGECEVRDSYPEGNVTWYKNGRVLQPVEEVNINLRKVENRSTGLFTMTSSLQYMPTKEDANAKFT CIVTYHGPSOQRTQ

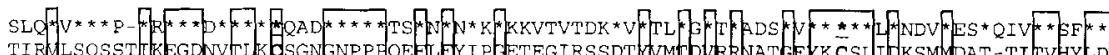

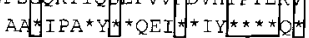

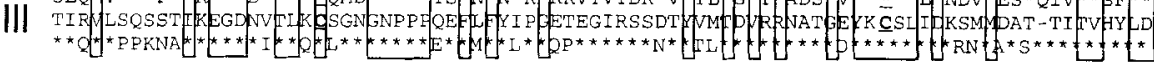

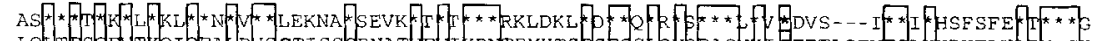

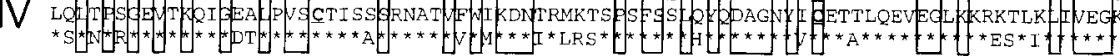

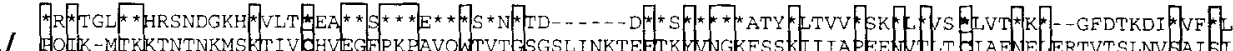

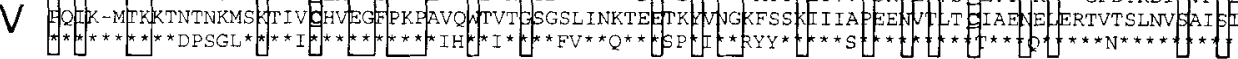

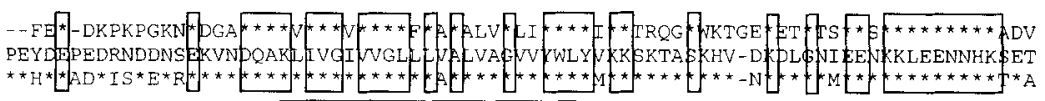

C

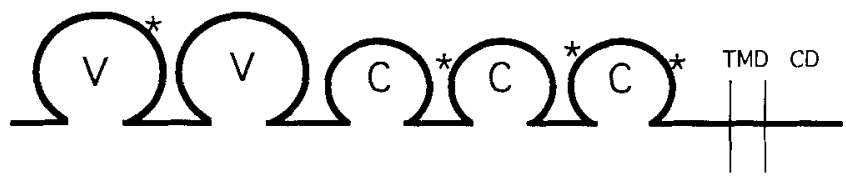

Figure 2 Zebrafish and mouse DM-GRASP are putative transmembrane glycoproteins and are members of the Immunoglobulin superfamily of cell adhesion proteins. (A) Kyte-Doolittle hydrophilicity plot of coding region of zebrafish DM-GRASP (window $=7$ amino acids). (B) Protein alignment (Geneworks) of zebrafish DM-GRASP and mouse DM-GRASP with chick DM-GRASP. Putative signal sequences are in bold italics, and transmembrane domains are underscored. The entire amino acid sequence of chick DM-GRASP is shown, while in the zebrafish and mouse sequences, only nonidentical amino acids are shown. Dashes in the zebrafish and mouse sequences represent identity with the aligned amino acids of the chick sequence. Boxed residues indicate amino acid identity between all three proteins. The sequences are aligned such that corresponding Ig domains (I-V) are identified. Each domain begins 20 amino acids before the first of two cysteine residues (bold and underscored) that define them (Williams and Barclay, 1988). Blank spaces indicate gaps inserted by the alignment program. The asterisk indicates the leucine residue substituted for the first cysteine in the fourth Ig domain of the zebrafish DM-GRASP sequence (see text). The mouse sequence has been registered with Genbank and given the following accession number: MUSDMGRASP, L25274. (C) Diagram illustrating the predicted protein structure of zebrafish DM-GRASP. V-type and C-type Ig domains are indicated along with potential N-linked glycosylation sites. Transmembrane (TMD) and cytoplasmic (CD) domains are indicated.

insert containing a $1071 \mathrm{bp}$ open reading frame followed by 215 bp of $3^{\prime}$ untranslated sequence. The sequence of this partial cDNA clone was aligned to the 3 ' portion of chick $D M-G R A S P$ (resi- dues 795-2,036) and was found to share $76 \%$ nucleotide identity (data not shown). The open reading frame of the mouse clone predicts a 357 amino acid protein which shares $76 \%$ identity with the 
carboxyl portion of the chick protein [Fig. 2(B)]. Comparison of protein subsequences between the mouse and chick clones indicates that over the extent of their partial alignment the mouse protein contains nearly all of the predicted structural features characteristic of chick DM-GRASP. These include a portion of the second V type Ig domain, three C2-type Ig domains, identical sites for potential asparagine-linked glycosylation, and a particularly high degree of homology in the predicted transmembrane domain $(95 \%)$ and cytoplasmic tail $(85 \%)$. The three-way alignment of the mouse clone with the carboxyl portions of both chick DM-GRASP and the zebrafish clone, indicates that they share $37 \%$ amino acid identity [Fig. 2(B)].

\section{Characterization of the Zebrafish DM-GRASP Protein}

A fusion protein containing 271 amino acids of the coding region of the zebrafish cDNA clone was used to generate antibodies for the characterization of the zebrafish protein. The fusion protein was purified by affinity chromatography and injected into three rabbits. All three rabbits produced antisera that reacted with the fusion protein while preimmune sera did not (data not shown). Immunoblots of lysates from 24- and 48-h zebrafish embryos were probed with the antisera raised against zebrafish DM-GRASP. All three antisera reacted with a common protein of approximately $72 \mathrm{kD}$ [Fig. 3(A)].

The discrepancy between the predicted molecular weight of the unprocessed core protein $(61 \mathrm{kD})$ and the apparent molecular weight detected by immunoblot analysis is presumed to be due to addition of carbohydrate moieties to the putative asparagine-linked glycosylation sites. To examine this, mRNA containing the entire protein coding region was transcribed and translated in vitro. The $\mathrm{mRNA}$ was translated in rabbit reticulocyte lysate in the presence and absence of canine pancreatic microsomal membranes, derived from ER. Proteins translated in the presence of purified microsomes should be cotranslationally modified and glycosylated. Proteins translated in the absence of membranes, on free ribosomes of the reticulocyte lysate should represent unmodified core proteins. Zebrafish DM-GRASP mRNA translated in reticulocyte lysate alone produced a core protein with an apparent molecular weight of $66 \mathrm{kD}$ [ Fig. 3(B)]. In contrast, the protein translated in the presence of microsomes had a higher molecular weight of approxi-

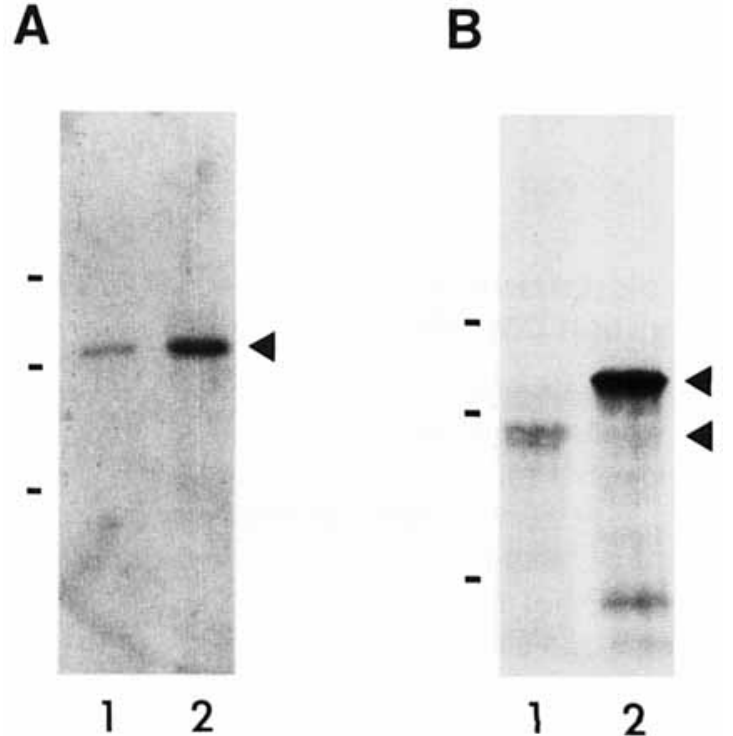

Figure 3 Analysis of the $72 \mathrm{kD}$ zebrafish DM-GRASP protein. (A) Immunoblots of zebrafish DM-GRASP (arrowhead) detected by UM28 antibodies. Lanes 1 and 2 represent embryo lysates from 24- and 48-h zebrafish embryos, respectively. Molecular weight markers are from top 97,68 , and $43 \mathrm{kD}$. (B) In vitro translation of zebrafish DM-GRASP mRNA using rabbit reticulocyte lysates (Promega). Lanes 1 and 2 represent proteins respectively translated in the absence (lower arrowhead) and presence (upper arrowhead) of canine microsomal membranes. Molecular weight markers are from top 97 , 68 , and $43 \mathrm{kD}$.

mately $78 \mathrm{kD}$. The lower molecular weight band in Figure 3(B) (lane 2) is presumably a degradation product.

The ability of zebrafish DM-GRASP protein translation to be directed to microsomal membranes, supports its classification as a putative transmembrane protein, and indicates that the core protein can undergo signal sequence processing and glycosylation. Since the observed $72 \mathrm{kD}$ zebrafish DM-GRASP protein is substantially larger than the core protein translated in vitro, glycosylation and signal sequence processing are likely to occur in vivo. The observation that the molecular weight of zebrafish DM-GRASP translated in the presence of microsomal membranes was larger $(78 \mathrm{kD})$ than that observed in vivo may be due to incomplete oligosaccharide processing by the in vitro system. High-mannose sugars are normally added to core proteins in the ER, with subsequent cleavage and modification occurring during vectorial transport through the golgi compartments (Hubbard and Ivatt, 1981; Kornfeld and Kornfeld, 1985). The $78 \mathrm{kD}$ protein translated in 
the presence of ER microsomes may represent a high-mannose precursor form of the mature $72 \mathrm{kD}$ zebrafish DM-GRASP glycoprotein expressed in vivo.

\section{Developmental Expression Pattern of Zebrafish DM-GRASP}

The antisera generated against the zebrafish DMGRASP fusion protein labeled a subset of neurons in the zebrafish embryo. The first cells to exhibit labeling were two classes of sensory neurons, the trigeminal ganglion neurons in the head and the Rohon-Beard neurons in the spinal cord. The trigeminal ganglion neurons extend efferent axons posteriorly into the hindbrain forming the lateral longitudinal fasciculus (LLF) (Metcalfe et al., 1990). The Rohon-Beard neurons extend their central axons anteriorly and posteriorly within the dorsolateral spinal cord to establish the dorsal longitudinal fasciculus (DLF). These cells exhibited weak labeling as early as $16 \mathrm{~h}$ which is approximately when they first extend axons (data not shown). By $24 \mathrm{~h}$, the cell bodies and central axons of trigeminal ganglion and Rohon-Beard neurons were well labeled [Fig. 4(A and B)]. The apparent cytoplasmic labeling seen in these neurons may represent the translation and processing of zebrafish DM-GRASP in the cell body. By this stage the descending trigeminal ganglion central axons and the ascending Rohon-Beard central axons have met and fasciculated to form a continuous longitudinal tract that was labeled by the antisera. [Fig. 4(C); $5(\mathrm{~A})$ ]. In addition, the neurons of the acousticvestibular nucleus were labeled at $24 \mathrm{~h}$ [Fig. 4(A)]. Thus, zebrafish DM-GRASP is expressed by a specific subset of axons that fasciculate with each other in the central nervous system (CNS).

Although there are at least eight other major tracts in the CNS at $24 \mathrm{~h}$ (Chitnis and Kuwada, 1990; Bernhardt et al., 1990), only the axons in the LLF/DLF expressed DM-GRASP. This selectivity of expression becomes more apparent when the pattern of labeling by anti-DM-GRASP antibodies is compared to that generated by an antibody against acetylated $\alpha$-tubulin, which labels virtually all axons in the zebrafish embryo (Chitnis and $\mathrm{Ku}$ wada, 1990). In the spinal cord the acetylated $\alpha$-tubulin antibody labeled axons in the DLF, commissural axons, axons in the ventral longitudinal fascicle (VLF) in the spinal cord, and motor axons at $24 \mathrm{~h}$ [Fig. 5(B)]. In contrast, antisera against DMGRASP labeled the central axons of Rohon-Beard
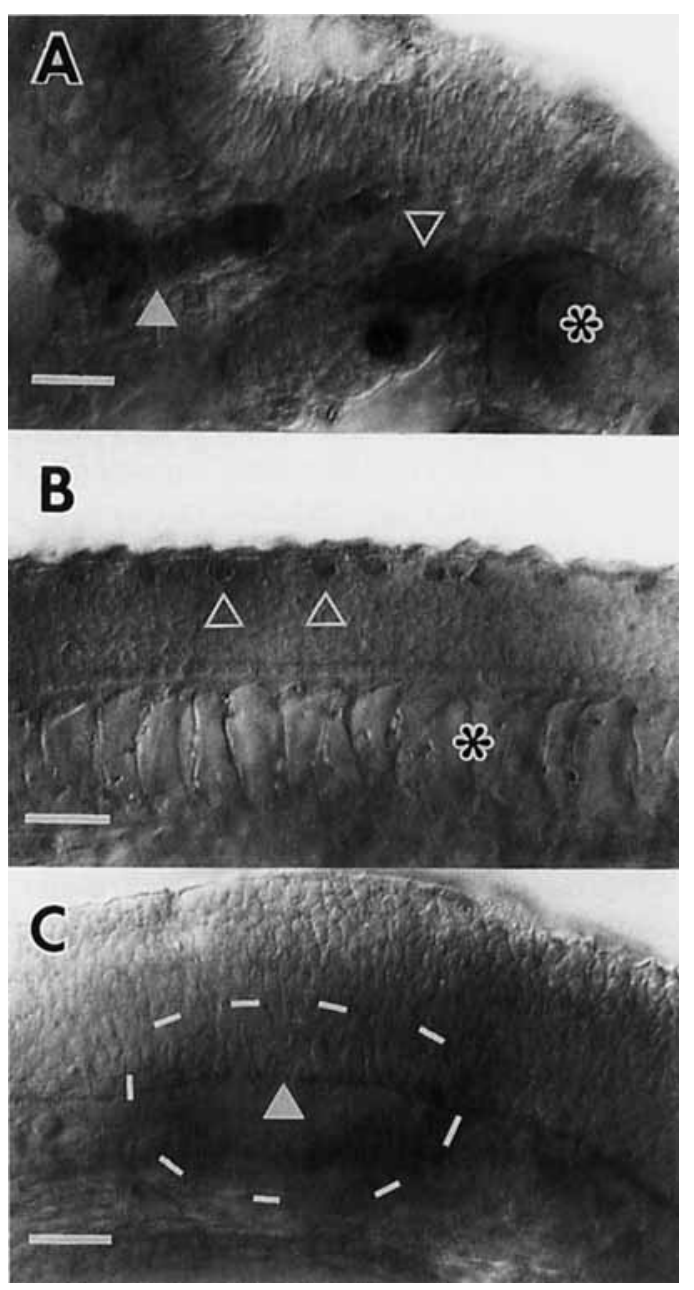

Figure 4 Zebrafish DM-GRASP is expressed by a single axon tract in the CNS at $24 \mathrm{~h}$. Whole-mount preparations of 24-h zebrafish embryos were incubated with affinity-purified antisera from rabbit UM5. Antibody labeling was detected by reaction with DAB. (A) Labeling of trigeminal ganglion (solid arrowhead) and acoustic-vestibular (open arrowhead) neurons in the zebrafish head. Asterisk indicates the otic vesicle. Scale bar $=30 \mu \mathrm{m}$. (B) Labeling of Rohon-Beard neurons (open arrowheads) in the spinal cord. Asterisk indicates the notochord. The row of floorplate cells just dorsal to the notocord is not labeled. Scale bar $=45 \mu \mathrm{m}$. (C) Labeling of the DLF and LLF in the region of the hindbrain where they first fasciculate to form a single longitudinal axon tract (arrowhead). Dashed circle indicates the otic vesicle out of the plane of focus. Scale bar $=30 \mu \mathrm{m}$. In all three panels anterior is to the left, and dorsal is upwards.

neurons, without labeling any of the other axons [Fig. 5(A)].

In addition to the selective expression in a single tract in the CNS at $24 \mathrm{~h}$, DM-GRASP expression is also restricted regionally on the trigeminal gan- 


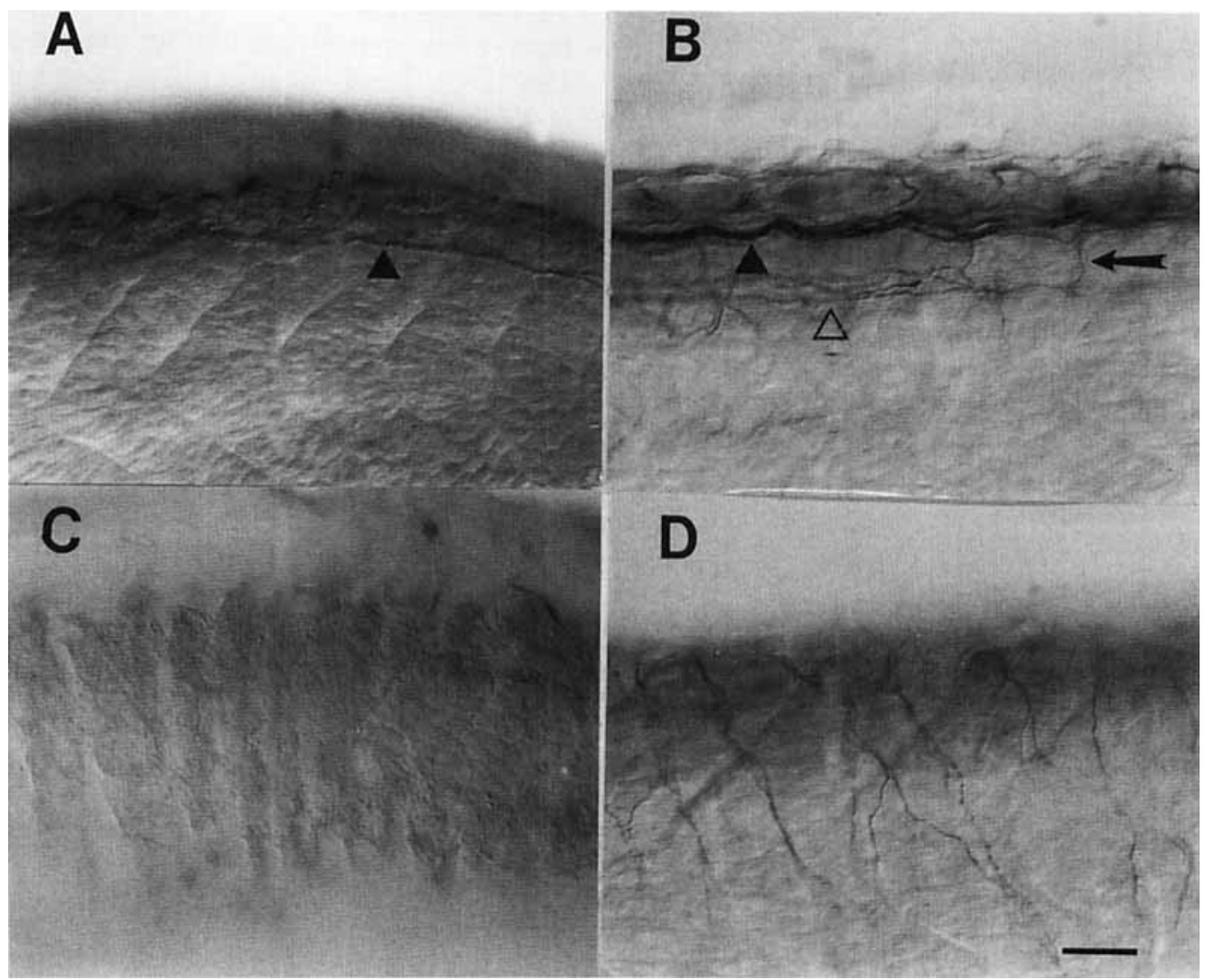

Figure 5 Zebrafish DM-GRASP is regionally expressed on specific axon domains of a subset of neurons. Whole-mount preparations of 24-h zebrafish embryos were incubated with affinity-purified antisera from rabbit UM5 ( $A$ and $C$ ), or a monoclonal antibody against acetylated $\alpha$-tubulin (B and D). (A) Labeling of the Rohon-Beard axons of the DLF (arrowhead) in the dorsal spinal cord. This embryo is tilted such that the dorsal and ventral spinal cord are not in the same plane of focus. At this stage there is no labeling in the ventral spinal cord. (B) Labeling of spinal axon tracts with the antibody against acetylated $\alpha$-tubulin labels the ventral longitudinal fascicle (VLF) (open arrowhead), and the DLF (closed arrowhead) which includes axons of Rohon-Beard cells, as well as the axons of commissural primary ascending (CoPA), ventral longitudinal descending (VeLD), and dorsal longitudinal ascending (DoLA) spinal interneurons. The arrow indicates a commissural axon of either a CoPA or CoSA interneuron. (C) Labeling of the skin over the spinal cord of an embryo labeled with UM5 antisera. Peripheral Rohon-Beard axons are devoid of labeling. (D) Labeling of the peripheral Rohon-Beard axons in the skin over the spinal cord of an embryo incubated with the antibody against anti-acetylated $\alpha$-tubulin. In all panels the magnification is the same as in (D). Scale bar $=30$ $\mu \mathrm{m}$.

glion and Rohon-Beard neurons. Trigeminal ganglion and Rohon-Beard neurons have central axons forming the LLF/DLF as well as peripheral axons that arborize extensively in the embryonic skin to innervate the head and body, respectively. Unlike the central axons that fasciculate with each other, the peripheral axons diverge from each other as they project distally. Although the central axons were labeled with the antisera against DMGRASP, the more distal branches of the peripheral axons were not [Fig. $5(\mathrm{C}) ;(6)]$. These peripheral axons were detected when labeled with the acetylated $\alpha$-tubulin antibody [Fig. 5(D)]. Thus regional expression of DM-GRASP on separate axonal domains of trigeminal ganglion and RohonBeard neurons is strongly correlated with fasciculation.

By $48 \mathrm{~h}$ in development, several other types of CNS neurons expressed DM-GRASP. The axons of retinal ganglion cells were prominently labeled 


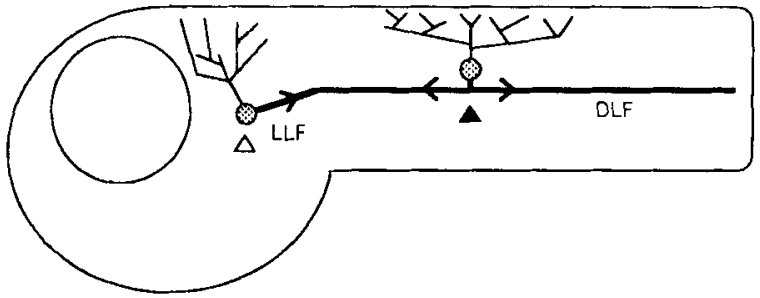

Figure 6 A diagram illustrating the relative positions and morphology of trigeminal ganglion neurons (open arrowhead) and Rohon-Beard neurons (closed arrowhead). Trigeminal ganglion neurons project central axons posteriorly forming the LLF while Rohon-Beard neurons project central axons bidirectionally forming the DLF. These central projections express Zebrafish DM-GRASP and fasciculate to form a single longitudinal axon tract. The trigeminal ganglion and RohonBeard neurons also project peripheral axons which arborize extensively in the skin. These peripheral axons do not express DM-GRASP.

as they projected from the retina towards the optic tectum [Fig. 7(A)]. DM-GRASP may also be regionally expressed on optic axons. Although optic axons innervate the anterior tectum by this time (data not shown), no prominent tectal labeling was observed. This expression pattern suggests that DM-GRASP is restricted to the fasciculated portions of optic axon domains, and is not expressed on the nonfasciculated arborizations within the tectum.

Several clusters of neurons in the rhombencephalon were labeled by the antisera at $48 \mathrm{~h}$ [Fig. $7(B)]$. The antisera labeled a large cluster of neurons located laterally along with a smaller dorsomedial cluster of neurons that extends axons ventro-medially to form a ventral commissure. Previous work suggests that these neurons are likely to be the commissural neurons that project their axons along the rhombomere segmental borders (Hanneman et al., 1988; Trevarrow et al., 1990). A small ventral cluster of neurons located next to the midline is also labeled with the antisera. These neurons may correspond to the motor nucleus of cranial nerve VI. Bilateral clusters of labeled neurons at the most anterior region of the rhombencephalon may correspond to presumptive cerebellar neurons and their afferent and/or efferent projections (data not shown). Examination of the cellular localization of labeling on these frozen sections demonstrates the association of zebrafish DMGRASP with the cell membranes of these neurons and supports its classification as a transmembrane protein (Fig. 7).
Although the axons and cell bodies of motoneurons in the spinal cord did not express zebrafish DM-GRASP at $24 \mathrm{~h}$, labeling was detected by $48 \mathrm{~h}$ (Fig. 8). The expression of zebrafish DM-GRASP on motoneuron axons was transient and by 5 days

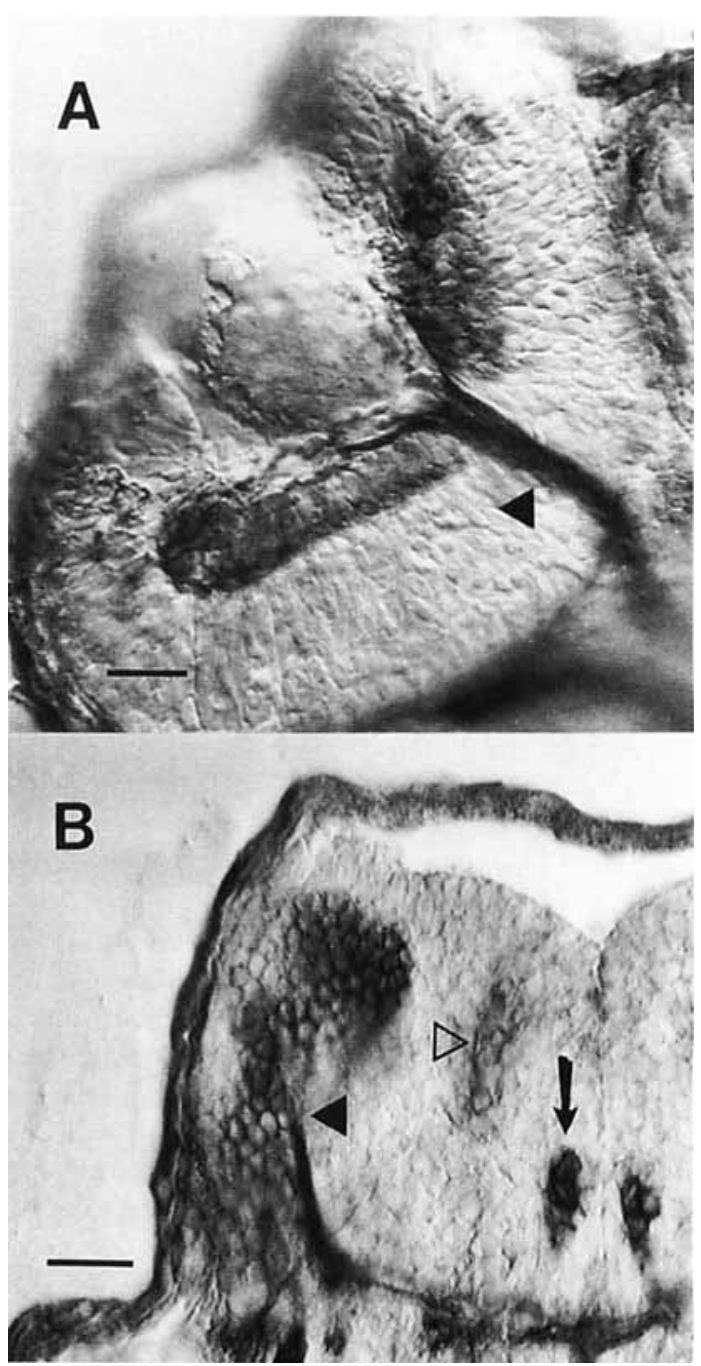

Figure 7 At 48 h Zebrafish DM-GRASP is expressed on the axon tracts of a several other neurons in the CNS. Frozen tissue sections of a 48 -h zebrafish embryo were incubated with affinity-purified antisera from rabbits UM27 and UM28. Antibody labeling was detected by reaction with $\mathrm{DAB}$. (A) A horizontal section through the head of the embryo showing the labeled retinal ganglion cells, and their labeled optic axons (arrowhead) exiting the eye and projecting towards the optic chiasm. Scale bar $=12 \mu \mathrm{m}$. (B) The left half of a cross-section through the zebrafish hindbrain shows a dorso-lateral cluster (solid arrowhead) and a smaller ventro-medial cluster (open arrowhead) of labeled neurons forming a ventral commissure. The arrow indicates the putative motor nucleus of cranial nerve VI. Scale bar $=17 \mu \mathrm{m}$. 


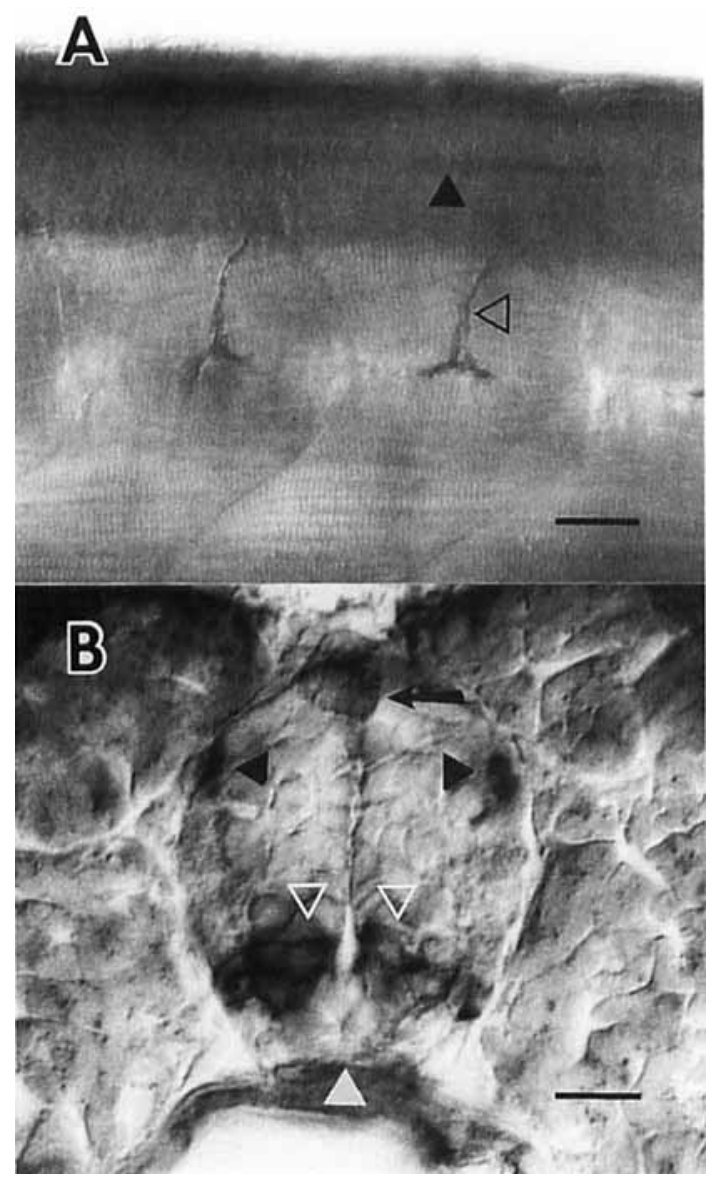

Figure 8 Motoneurons are labeled by affinity-purified antisera at $48 \mathrm{~h}$. Zebrafish embryos have been incubated with antisera from rabbits UM27 and UM28. (A) A whole-mount preparation with anterior to the right and dorsal upwards. The motoneurons exit the ventral spinal cord and travel along the medial surface of the myotome to the medial septum (open arrowhead). The DLF can be seen slightly out of the plane of focus (solid arrowhead). Scale bar $=20 \mu \mathrm{m}$. (B) Frozen tissue cross-section through the spinal cord of the embryo with dorsal upwards. Labeled bilateral clusters represent motoneurons (open arrowheads) in the ventral spinal cord. A Rohon-Beard neuron (arrow) and the DLF (black arrowheads) are labeled in the dorsal spinal cord. The floorplate cells located between the motoneuron clusters at the ventral midline (white arrowhead) are devoid of labelling. Scale bar $=11 \mu \mathrm{m}$.

motoneuron labeling was drastically reduced except for residual labeling in the muscle fibers (data not shown). The residual labeling in the muscles may correspond to motoneuron terminals. Transient expression of DM-GRASP was also observed on chick motoneurons (Pourquie et al., 1990).

Other labeled structures included the sensory cells of the otic vesicle, the anterior lateral line, and, to a lesser extent, the posterior lateral line. Less prominent labeling of the notochord and the nephric tubules was also observed. Unlike the chick, the floorplate cells found at the ventral midline of the spinal cord did not exhibit labeling [Figs. 4(B); 8(B)] (Burns et al., 1991). Table 1 briefly summarizes the expression patterns of DMGRASP in the zebrafish and the chick (Tanaka and Obata, 1984; Pourquie et al., 1990; Burns et al., 1991; El Deeb et al., 1992).

\section{DISCUSSION}

We have isolated and characterized a full-length zebrafish cDNA clone and a partial mouse cDNA clone that are similar to chick DM-GRASP. The nucleotide sequence of the zebrafish clone shares $54 \%$ identity, and predicts $39 \%$ amino acid identity, with chick DM-GRASP. This degree of sequence identity between distantly related species is within the range of other known homologs (Grenningloh et al., 1990). The conservation of Ig domain types and sequence (VVCCC) along with the similarity of expression patterns suggest that the zebrafish cDNA clone and chick DM-GRASP are homologs. The nucleotide sequence of the partial mouse clone shares $76 \%$ identity, and predicts $76 \%$ amino acid identity, with the carboxyl terminal portion of chick DM-GRASP. This high degree of sequence homology suggests that it represents a mammalian homolog of chick DM-GRASP. Although the amino terminal end of the mouse clone containing the $\mathrm{V}$-type Ig domains has not been isolated, conservation of the three C2-type Ig and cytoplasmic domains in all three of these proteins suggests that they, and possibly Neurolin in the goldfish (Paschke et al., 1992), may represent a distinct subfamily within the Ig superfamily of cell adhesion molecules.

The predicted amino acid sequence of zebrafish DM-GRASP, posttranslational processing in vitro, and association with the cell membranes of neurons suggest that it is a transmembrane glycoprotein. The conservation of extracellular Ig domains (VVCCC) and similar patterns of expression suggest that the extracellular domains of these proteins may serve similar developmental functions in both species. Chick DM-GRASP binds homophilically in vitro and promotes neurite outgrowth in vitro (Tanaka et al., 1991; Burns et al., 1991). These properties suggest that chick DM-GRASP mediates selective fasciculation of axons, and this may 
Table 1 Comparison of DM-GRASP Expression in Chick and Zebrafish Embryos

\begin{tabular}{|c|c|}
\hline Chick DM-Grasp & Zebrafish DM-Grasp \\
\hline Dorsal root ganglia sensory neurons & Rohon-Beard sensory neurons \\
\hline Trigeminal neurons & Trigeminal neurons \\
\hline Dorsal funiculus of spinal cord & $\begin{array}{l}\text { Dorsal lateral fascicle (DLF)/Lateral longitudinal } \\
\text { fascicle (LLF) }\end{array}$ \\
\hline Motoneurons & Motoneurons \\
\hline Ventral funiculus of spinal cord/ventral horn & Ventral motor column/ventral motor roots \\
\hline Cranial motoneurons & Cranial motoneurons \\
\hline . & Lateral line sensory neurons \\
\hline Proximal nucleus of Acoustic-vestibular/ facial neurons & Acoustic-vestibular neurons \\
\hline- & Retinal ganglia neurons \\
\hline$C_{-1}$ & Hindbrain commissural neurons \\
\hline Sympathetic neurons & - \\
\hline Inferior olivocerebellar neurons & $\begin{array}{l}\text { Afferent/efferent projections to presumptive } \\
\text { cerebellum (?) }\end{array}$ \\
\hline Floorplate cells of spinal cord & - \\
\hline Notochord & Notochord \\
\hline Nephric tubules & Nephric tubules \\
\hline Epidermis & - \\
\hline
\end{tabular}

also be true of zebrafish DM-GRASP (see below). Aithough the expression of chick DM-GRASP has been observed on growth cones (Pourquie et al., 1992), the localization of zebrafish DM-GRASP on extending growth cones could not be determined conclusively, and is presently under investigation.

The highest degree of homology between zebrafish, mouse, and chick DM-GRASP is in their transmembrane and cytoplasmic regions. The conservation of these domains may indicate a necessary and common function of these intracellular domains. Transmembrane proteins are capable of interacting with cytoskeletal elements or transducing extracellular information to specific intracellular signals through specialized cytoplasmic domains. For example, the Drosophila LAR protein is a member of the Ig family containing both extracellular Ig domains and cytoplasmic tyrosine phosphatase domains ( Streuli et al., 1989). Neuroglian, another Ig family member, has multiple forms containing identical extracellular domains but different cytoplasmic domains. These multiple forms are selectively expressed in different cell types suggesting distinct cellular functions (Hortsch et al., 1990). Although it is not known what role chick, mouse, and zebrafish DM-GRASP play in signal transduction, the high level of conservation of their transmembrane and cytoplasmic domains supports the possibility that they serve necessary and similar intracellular functions.

DM-GRASP may guide growth cones to their targets in the zebrafish CNS by mediating selective fasciculation of the axons that express it. Like chick DM-GRASP, the zebrafish homolog is expressed on a subset of axons. At $24 \mathrm{~h}$, expression was restricted to the axons forming the LLF in the hindbrain and the DLF in the spinal cord. The central axons of the trigeminal ganglion and RohonBeard neurons fasciculate with each other in the CNS establishing a single continuous longitudinal pathway (LLF/DLF). Both sets of neurons begin expressing DM-GRASP at about the time they initially project axons. This expression pattern, and the fact that chick DM-GRASP binds homophilically, suggest that the proper formation of the LLF/DLF and guidance of trigeminal ganglion and Rohon-Beard axons in the CNS may depend on DM-GRASP.

While the fasciculated central axons of the trigeminal ganglion and Rohon-Beard neurons expressed zebrafish DM-GRASP, their nonfasciculated peripheral axons were conspicuously devoid of antibody labeling. The lack of zebrafish DMGRASP expression in these peripheral axons may account for their defasciculation as they branch extensively throughout the skin. Zebrafish DMGRASP was also expressed regionally on the axons of the retinal ganglion cells. The expression of zebrafish DM-GRASP was limited to regions where optic axons fasciculate with each other as they extend towards the tectum. These axons did not express zebrafish DM-GRASP where they defasciculate and arborize within the tectum. These obser- 
vations further support the potential role of zebrafish DM-GRASP in the fasciculation of specific axons.

Other Ig family members, such as fasciclin II in the grasshopper and $\mathrm{Ll}$ in the rat, are regionally expressed on fasciculated axon domains (Goodman et al., 1985; Harrelson and Goodman, 1988; Dodd et al., 1988; Schachner et al., 1990). Commissural neurons projecting axons across the midline increase their expression of these surface molecules when they change course and specifically fasciculate with contralateral longitudinal axon tracts. In the chick, the cell surface molecule Bravo is regionally expressed on optic axons in a pattern similar to zebrafish DM-GRASP (De La Rosa et al., 1990). Retinal ganglion cells express Bravo on optic axons within the retina, but not on their axon domains within the optic tectum. The domain-specific expression of these molecules, including zebrafish DM-GRASP, suggests that these proteins are responsible for guiding growth cones along different portions of their pathway.

The expression of zebrafish DM-GRASP on motor axons is transient, as it is in the chick (Tanaka and Obata, 1984; Pourquie et al., 1990). This temporal regulation suggests that zebrafish DMGRASP plays a developmental role during axonogenesis, and that subsequent expression is not necessary for the maintenance of these axonal pathways. Primary motoneurons pioneer the motor axon pathway between 15 and $18 \mathrm{~h}$, while secondary motoneurons project axons between 24 and 48 h (Myers et al., 1986; Westerfield et al., 1986; Eisen et al., 1986). Zebrafish DM-GRASP was not evident in motoneurons before $24 \mathrm{~h}$, and, therefore, correlates with the axonogenesis of secondary motoneurons. This is consistent with the observation that trigeminal ganglion and Rohon-Beard neurons also begin expressing DM-GRASP at the time of initial axon extension.

A potential goldfish homolog of DM-GRASP, Neurolin, is also correlated with new axon outgrowth (Paschke et al., 1992). Neurolin exhibits both a comparable pattern of expression in the spinal cord, and nucleotide sequence homology to chick DM-GRASP (Laessing et al., 1993) and is likely to be a homolog of zebrafish DM-GRASP. The expression of Neurolin may correlate with new axon outgrowth, and the regeneration of ganglion cells within the marginal growth zone of the retina and the optic tectum (Paschke et al., 1992). Although zebrafish DM-GRASP and Neurolin are both expressed on specific axon domains of retinal ganglion cells, their expression patterns are not en- tirely conserved. Unlike Neurolin, zebrafish DMGRASP is expressed throughout the ganglion cell layer in the retina, and does not appear to be expressed in the optic tectum. Furthermore zebrafish DM-GRASP is not expressed in the floorplate of the spinal cord as it is in the goldfish and the chick (Tanaka et al., 1991; Burns et al., 1991; Paschke et al., 1992).

We have described the cloning and initial characterization of a full-length zebrafish cDNA and a partial mouse cDNA similar to chick DMGRASP. We have taken advantage of the simple and highly characterized nervous system of the zebrafish embryo to examine the expression of zebrafish DM-GRASP. The zebrafish DM-GRASP protein is dynamically expressed during axonogenesis on sensory and motor axons of the developing embryo. Its selective pattern of expression on specific axon domains correlates with fasciculation and suggests a role in guiding axons. Further characterization of this putative axon guidance molecule and the manipulation of its expression in transgenic zebrafish (Weinberg, 1992) should lead to a better understanding of its role in directing axonal outgrowth in vivo.

The authors would like to thank Dr. K. Zinn and Dr. D. Grunwald for supplying the zebrafish cDNA libraries and Dr. A. Saltiel for the generous use of his laboratory facilities for the biochemical analysis in this work. We thank V. Abatzis for technical assistance with the immunocytochemical analysis and D. Bay for photographic work. We would also like to thank Dr. K. Milarski and Dr. S. Kwak for helpful discussions and comments on the manuscript. J.P.K. was supported by an NIH postdoctoral fellowship. This work was supported by NIH grants NS24848 to J.Y.K. and NS26519 to S.C.

\section{REFERENCES}

Bastiani, M. J., DU-LaC, S., and Goodman, C. S. (1986). Guidance of neuronal growth cones in the grasshopper embryo. I. Recognition of a specific axonal pathway by the pCC neuron. J. Neurosci. 6:3518-3531.

Bastiani, M. J., Harrelson, A. L., SnOw, P. M., and Goodman, C. S. (1987). Expression of fasciclin I and II glycoproteins on subsets of axon pathways during neuronal development in the grasshopper. Cell, 48:745-755.

BENTLEY, D. and CAUDY, M. (1983). Navigational substrates for peripheral pioneer growth cones: limb-axis polarity cues, limb-segment boundaries, and guidepost neurons. Cold Spring Harb. Symp. Quant. Biol. 2:573-585. 
Bernhardt, R. R., Chitnis, A. B., Lindamer, L., and KUWADA, J. Y. (1990). Identification of spinal neurons in the embryonic and larval zebrafish. J. Comp. Neurol. 302:603-616.

Burns, F. R., von-KanNen, S., GuY, L., RAPER, J. A., KAMHOLZ, J., and CHANG, S. (1991). DM-GRASP, a novel immunoglobulin superfamily axonal surface protein that supports neurite extension. Neuron 7:209-220.

Chitnis, A. B. and Kuwada, J. Y. ( 1990). Axonogenesis in the brain of zebrafish embryos. $J$. Neurosci. 10(6): 1892-1905.

De la Rosa, E. J., Kayyem, J. F., Roman, J. M., StierHOF, Y. D., DREYER, W. J., and SCHWARZ, U. ( 1990 ). Topologically restricted appearance in the developing chick retinotectal system of Bravo, a neural surface protein: experimental modulation by environmental cues. J. Cell Biol. 111:3087-3096.

DodD, J. and Jessell, T. M. (1988). Axon guidance and the patterning of neuronal projections in vertebrates. Science 242:692-699.

Dodd, J., Morton, S. B., Karagogeos, D., YamaMOTO, M., and JeSSELl, T. M. ( 1988 ). Spatial regulation of axonal glycoprotein expression on subsets of embryonic spinal neurons. Neuron 1:105-116.

EISEN, J. S. (1991). Developmental neurobiology of the zebrafish. J. Neurosci. 11:311-317.

Eisen, J. S., Myers, P. Z., and Westerfield, M. (1986). Pathway selection by growth cones of identified motoneurones in live zebra fish embryos. Nature 320:269-271.

El Deeb, S., Thompson, S. C., and Covault, J. (1992). Characterization of a cell surface adhesion molecule expressed by a subset of developing chick neurons. Dev. Biol. 149:213-227.

Goodman, C. S., Bastiani, M. J., DoE, C. Q., and DuLAC, S. (1985). Growth cone guidance and cell recognition in insect embryos. In: Developmental Biology: A Comprehensive Synthesis, vol. 3. M. S. Steinberg, Ed. Plenum, New York, pp. 283-300.

Grenningloh, G., Bieber, A. J., ReHM, E. J., SNow, P. M., Traquina, Z. R., Hortsch, M., Patel, N. H., and GoOdMAN, C.S. ( 1990). Molecular genetics of neuronal recognition in Drosophila: evolution and function of immunoglobulin superfamily cell adhesion molecules. Cold Spring Harb. Symp. Quant. Biol. 55:327-340.

HAKES, D. J. and Dixon, J. E. ( 1992). New vectors for high level expression of recombinant proteins in bacteria. Anal. Biochem. 202:293-298.

Hanneman, E., Trevarrow, B., Metcalfe, W. K., KimMEL, C. B., and Westerfield, M. (1988). Segmental pattern of development of the hindbrain and spinal cord of the zebrafish embryo. Development 103:49-58.

HaRlow, E. and LANE, D. (1988). Antibodies: A Laboratory Manual. Cold Spring Harbor Laboratory: Cold Spring Harbor Laboratory, New York.
Harrelson, A. L. and Goodman, C. S. (1988). Growth cone guidance in insects: fasciclin II is a member of the immunoglobulin superfamily. Science 242:700-707.

Harris, W. A., Holt, C. E., and Bonhoeffer, F. (1987). Retinal axons with and without their somata, growing to and arborizing in the tectum of Xenopus embryos: a time-lapse video study of single fibres in vivo. Development 101:123-133.

Hortsch, M., Bieber, A. J., PATEL, N. H., and GoodMAN, C. S. (1990). Differential splicing generates a nervous system-specific form of Drosophila neuroglian. Neuron 4:697-709.

HubBard, S. C. and IVATT, R. J. (1981). Synthesis and processing of asparagine-linked oligosaccharides. Ann. Rev. Biochem. 50:555-583.

KAPFHAMmer, J. P. and RAPER, J. A. (1987). Collapse of growth cone structure on contact with specific neurites in culture. J. Neurosci. 7:201-212.

Kimmel, C. B., Powell, S. L., and Metcalfe, W. K. (1982). Brain neurons which project to the spinal cord in young larvae of the zebrafish. J. Comp. Neurol. 205:112-127.

KORNFELD, R. and KORNFELD, S. (1985). Assembly of asparagine-linked oligosaccharides. Ann. Rev. Biochem. 54:631-664.

KuWADA, J. Y. (1986). Cell recognition by neuronal growth cones in a simple vertebrate embryo. Science 233:740-746.

KuWADA, J. Y. and BERNHARDT, R. R. ( 1990). Axonal outgrowth by identified neurons in the spinal cord of zebrafish embryos. Exp. Neurol. 109:29-34.

Laessing, U., Giordano, S., LotTSPeich, F., and STUERMER, C. A. O. (1993). Molecular cloning of Neurolin and its expression in goldfish and embryonic zebrafish CNS. Soc. Neurosci. Abstr. 19:1090.

LeHmanN, J. M., Riethmuller, G., and Johnson, I. P. (1989). MUC18, a marker of tumor progression in human melanoma, shows sequence similarity to the neural cell adhesion molecules of the immunoglobulin superfamily. Proc. Natl. Acad. Sci. U.S.A. 86:9891-9895.

Metcalfe, W. K., Myers, P. Z., Trevarrow, B., BaSS, M. B., and KIMMEL, C. B. (1990). Primary neurons that express the $\mathrm{L} 2$ / HNK- 1 carbohydrate during early development in the zebrafish. Development 110:491-504.

MYERS, P. Z. (1985). Spinal motoneurons of the larval zebrafish. J. Comp. Neurol. 236:555-561.

MYers, P. Z., EISEN, J. S., and Westerfield, M. (1986). Development and axonal outgrowth of identified motoneurons in the zebrafish. J. Neurosci. 6:2278-2289.

Paschke, K. A., LotTspeich, F., and Stuermer, C. A. (1992). Neurolin, a cell surface glycoprotein on growing retinal axons in the goldfish visual system, is reexpressed during retinal axonal regeneration. $J$. Cell Biol. 117:863-875.

Pearson, W. R. and LipMan, D. J. (1988). Improved 
tools for biological sequence comparison. Proc. Natl. Acad. Sci. U.S.A. 85:2444-2448.

Pourquie, O., Coltey, M., Thomas, J. L., and LeDOUARIN, N. M. (1990). A widely distributed antigen developmentally regulated in the nervous system. $D e-$ velopment 109:743-752.

Pourquie, O., Corbel, C., Le-Caer, J. P., Rossier, J., and LE-DOUARIN, N. M. (1992). BEN, a surface glycoprotein of the immunoglobulin superfamily, is expressed in a variety of developing systems. Proc. Natl. Acad. Sci. U.S.A. 89:5261-5265.

Raper, J. A., Bastiani, M., and Goodman, C. S. (1983). Pathfinding by neuronal growth cones in grasshopper embryos. II. Selective fasciculation onto specific axonal pathways. $J$. Neurosci. 3:31-41.

RAthJeN, F. G. and JESSElL, T. M. (1991). Glycoproteins that regulate the growth and guidance of vertebrate axons: domains and dynamics of the immunoglobulin/fibronectin type III subfamily. In: Sem. in Neurosci. Harcourt Brace Jovanovich, London, England, pp. 297-307.

Rathjen, F. G., WolfF, J. M., Frank, R., BonHOEFFER, F., and Rutishauser, U. (1987). Membrane glycoproteins involved in neurite fasciculation. J. Cell Biol. 104:343-353.

ReIChardT, L. F. and Tomaselli, K. J. (1991). Extracellular matrix molecules and their receptors: functions in neural development. Annu. Rev. Neurosci. 14:531-570.

Saiki, R. K., Gelfand, D. H., StofFel, S. Scharf, S. J., HiguchI, R., HoRN, G. T., Mullis, K. B., and ERLICH, H. A. (1988). Primer-directed enzymatic amplification of DNA with a thermostable DNA polymerase. Science 239:487-491.

Sambrook, J., Fritsch, E, F., and Maniatis, T. (1989). Molecular Cloning: A Laboratory Manual. Cold Spring Harbor Laboratory, Cold Spring Harbor, New York.

SANGer, F., Nicklen, S., and Coulson, A. R. (1977). DNA sequencing with chain-terminating inhibitors. Proc. Natl. Acad. Sci. U.S.A. 74:5463-5467.

SCHACHNER, M., ANTONICEK, H., FAHRIG, T., Faissner, A., Fischer, G., Kunemund, V., MARtini, R., Meyer, A., Persohn, E., Pollerberg, E., Probstmeier, R., Sadoul, K., Sadoul, R., SeilHEIMER, B., and THOR, G. (1990). Families of neural cell adhesion molecules. In: Morphoregulatory Mole- cules. G. M. Edelman, B. A. Cunningham, and J. P. Thierry, Eds. John Wiley and Sons, New York, pp. 443-468.

Smith, D. B. and Johnson, K. S. (1988). Single-step purification of polypeptides expressed in Escherichia coli as fusions with glutathione S-transferase. Gene $67: 31-40$.

Streuli, M., Krueger, N. X., TSAI, A. Y., and SaIto, H. (1989). A family of receptor-linked protein tyrosine phosphatases in humans and Drosophila. Proc. Natl. Acad. Sci. U.S.A. 86:8698-8702.

Tanaka, H., Matsui, T., Agata, A., Tomura, M., Kubota, I., McFarland, K. C., KoHR, B., LeE, A., Phillips, H. S., and Shelton, D. L. (1991). Molecular cloning and expression of a novel adhesion molecule, SC1. Neuron 7:535-545.

TANAKA, H. and OBATA, K. (1984). Developmental changes in unique cell surface antigens of chick embryo spinal motoneurons and ganglion cells. Dev. Biol. 106:26-37.

Tessier-Lavigne, M. and Placzek, M. (1991). Target attraction: are developing axons guided by chemotropism? Trends Neurosci. 14:303-310.

TOSNEY, K. W. and LANDMESSER, L. T. (1985). Growth cone morphology and trajectory in the lumbosacral region of the chick embryo. J. Neurosci. 5:2345-2358.

Trevarrow, B., Marks, D. L., and Kimmel, C. B. (1990). Organization of hindbrain segments in the zebrafish embryo. Neuron 4:669-679.

voN HEIJNE, G. (1986). A new method for predicting signal sequence cleavage sites. Nucleic Acids Res. 14:4683-4690.

WEINBERG, E. S. (1992). Analysis of early development in the zebrafish embryo. Results Probl. Cell Differ. 18:91-150.

WesterfiELD, M. (1993). The Zebrafish Book. University of Oregon Press, Eugene, OR.

Westerfield, M., MCMurray, J. V., and Eisen, J. S. (1986). Identified motoneurons and their innervation of axial muscles in the zebrafish. $J$. Neurosci. 6:22672277.

Williams, A. F. and Barclay, A. N. (1988). The immunoglobulin superfamily-domains for cell surface recognition. Annu. Rev. Immunol. 6:381-405.

Wilson, S. W. and EASTER, S., JR. ( 1991). Stereotyped pathway selection by growth cones of early epiphysial neurons in the embryonic zebrafish. Development 112:723-746. 